\title{
Soleamiento e iluminación natural en la edificación siguiendo un modelo conjunto de clase invertida y resolución de problemas
}

\section{Sun path and natural lighting in buildings following a joint model of flipped classroom and problem-based learning}

ANTONIO SERRANO-JIMÉNEZ

ORCID: http://orcid.org/0000-0002-8585-857X

Universidad de Sevilla

Departamento de Construcciones

Arquitectónicas I

aserrano5@us.es

Fecha de recepción:

Fecha de aceptación:

DOI: http://dx.doi.org/10.12795/9788447221912.070

Pp.: 1615-1639 
La formación del arquitecto pasa por reconocer la importancia del entorno en el que se sitúa un edificio para optimizar su emplazamiento, orientación, apertura de huecos o técnicas pasivas incorporadas en su envolvente. Esta comunicación presenta un Ciclo de Mejora en el Aula en contenidos de acondicionamiento lumínico y térmico de la asignatura de Construcción I, del primer curso del Grado en Fundamentos de la Arquitectura de la Universidad de Sevilla, implantando un nuevo modelo docente que combina la resolución de problemas con la clase invertida que permite alcanzar un aprendizaje mucho más efectivo a través de la resolución de ejercicios prácticos en el aula, el razonamiento en grupo y su aplicación en la realidad. La propuesta docente incorpora nuevas técnicas y recursos didácticos, así como también herramientas para su evaluación, obteniendo resultados positivos en la satisfacción y el aprendizaje adquirido por los estudiantes.

Palabras clave: Construcción I, Arquitectura, Docencia universitaria, Experimentación docente universitaria, Aula invertida.

\section{Abstract}

The architect's training involves recognising the importance of the environment in which a building is located to optimise its location, orientation, opening of hollows or passive techniques incorporated in its envelope. This communication presents an Improvement cycle in Classroom related to the lighting and thermal conditioning contents in the subject of Construction I, that belongs to the first year of the Fundamentals of Architecture Degree of the University of Seville, implementing a new teaching model that combines the problem-based learning with the flipped classroom that allows to achieve more effective learning through the resolution of practical exercises in the classroom, group reasoning and its application to reality. The teaching proposal incorporates new techniques and didactic resources, as well as tools for its evaluation, obtaining positive results in the satisfaction and learning acquired by the students.

Keywords: Construction I, Architecture, University teaching, University teaching experimentation, Flipped classroom.

Jornadas de Formación e Innovación Docente del Profesorado | № 2 (2019) Esta obra se distribuye con la licencia Creative Commons Reconocimiento-NoComercial-SinObraDerivada Internacional (CC BY-NC-ND 4.0.) 


\section{Descripción del contexto de la asignatura}

La asignatura de Construcción I es una asignatura obligatoria del primer cuatrimestre, que consta de 6 créditos ECTS y que se imparte en el primer curso del Grado en Fundamentos de la Arquitectura. El objetivo de la asignatura es el de introducir los conocimientos fundamentales y dar una visión global de los sistemas y técnicas constructivas que existen en las diferentes fases de construcción de un edificio, así como unas nociones básicas sobre los factores que influyen el acondicionamiento y el confort interior. La organización del curso se estructura en dos bloques fundamentales, sistemas constructivos y acondicionamiento del edificio, cada uno de los cuales se imparte a través de diferentes temas independientes, con una parte de teoría y práctica. La base de conocimiento previo requerida en este bloque se fundamenta en conocimientos previos de Dibujo Técnico, con una importancia de la visión espacial y la representación gráfica, y algunos conceptos de la Física y las Matemáticas.

Esta comunicación presenta el Ciclo de Mejora en el Aula (CIMA) aplicado entre los meses de septiembre y octubre de 2019 a lo largo de 12 horas, repartidas en seis sesiones de dos horas cada una, en un grupo de 29 alumnos mayoritariamente de nuevo ingreso, aunque existe un $20 \%$ de esos alumnos que están repitiendo la asignatura. Los contenidos que se van a tratar en este CIMA pertenecen al bloque de acondicionamiento lumínico y térmico del edificio, relativo al estudio del soleamiento, la iluminación natural y el diseño de técnicas de acondicionamiento pasivo de la envolvente del edificio, que garanticen el confort y el bienestar de los ocupantes de una forma sostenible (Figura 1).

Jornadas de Formación e Innovación Docente del Profesorado | № 2 (2019) Esta obra se distribuye con la licencia Creative Commons Reconocimiento-NoComercial-SinObraDerivada Internacional (CC BY-NC-ND 4.0.) 
CONSTRUCCIÓN 1

Bloques de contenidos
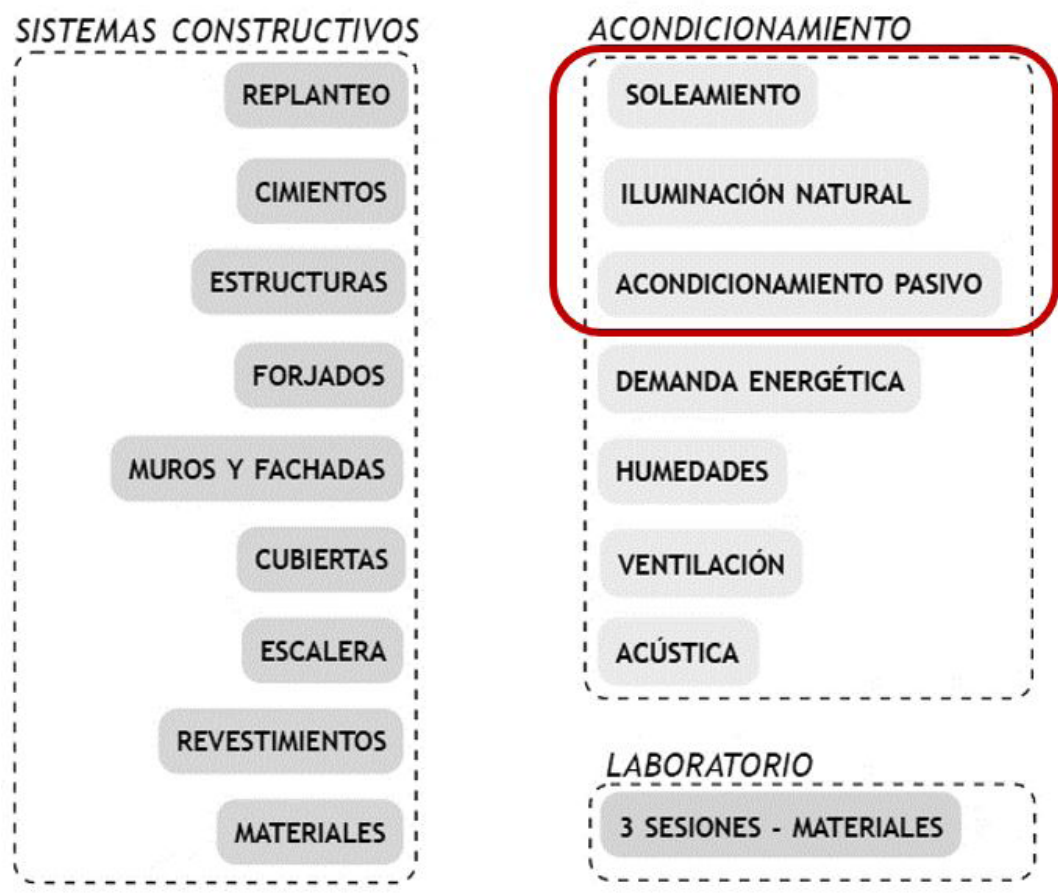

Figura 1. Organización de la asignatura y contenidos seleccionados para el CIMA.

Durante los últimos años, las clases se constituían principalmente mediante sesiones teóricas apoyadas en el uso principalmente del PowerPoint y, con una breve explicación práctica del profesor, el alumno tenía que resolver en casa ejercicios prácticos de una mayor dificultad a la enseñada. Este procedimiento ofrecía fundamentalmente resultados negativos y un aprendizaje no eficaz, que provocaba que los alumnos no razonasen la resolución de ejercicios y que memorizasen los diferentes contenidos tratados en cada bloque temático. Además, se generaba una carencia de identificación de la teoría con la realidad, que podría ser perfectamente visible en vídeos, fotografias o, en un paseo por la calle o una visita a una 
obra. Por último, al ser la primera toma de contacto de los alumnos con ciertos materiales y las técnicas de construcción, resultaba necesario que la docencia se acompañara con la introducción a ciertas competencias profesionales, a la resolución efectiva de problemas, a un aprendizaje efectivo que incite al razonamiento del alumno o a desarrollar la capacidad de comprensión gráfica o visión espacial.

\section{Diseño del CIMA}

Durante los años anteriores se ha contado con la experiencia de desarrollar un ciclo de innovación docente en la misma asignatura en el bloque temático del acondicionamiento térmico en el edificio, fundamentado en un aprendizaje basado en problemas, que hiciera a los alumnos razonar y aprender los conceptos fundamentales que influyen en garantizar un confort térmico en la edificación y saber decidir la elección de materiales con diferentes capacidades aislantes en el diseño de las capas de la envolvente térmica del edificio (Krauss y Boss, 2013; Serrano-Jiménez et al., 2017). También se ha incorporado en otros ciclos nuevos recursos digitales como el uso de vídeos, tutoriales y nuevos esquemas gráficos para comprender la función y utilidad de cada una de las capas que componen las cubiertas plana e inclinada de los edificios, obteniendo unos resultados más exitosos en los ejercicios prácticos, y sobre todo una gran satisfacción (87\%) expresada por los alumnos en el cuestionario sobre la opinión del ciclo de mejora implantado (Bain, 2007). Ambos ciclos han demostrado en los resultados alcanzados que ciertos retoques y mejoras puntuales en la docencia pueden suponer un salto del porcentaje situado en cada escalón de la escalera de aprendizaje, con una ampliación del 10-20\% en los últimos escalones. 
Los temas tratados en este CIMA ofrecen un primer avance de la importancia de las condiciones exteriores de iluminación natural para la orientación, emplazamiento, apertura de huecos y diseño de técnicas pasivas en la envolvente de un edificio, en función de las zonas climáticas en la que se trabaje. Cada uno de estos temas y su interrelación componen un conocimiento fundamental en la formación del arquitecto y para el alumno de nuevo ingreso resulta compleja la comprensión de este bloque a través de la enseñanza tradicional, que provoca la mecanización y memorización, en muchas ocasiones errónea, de un fenómeno natural que podría percibirse y razonarse en múltiples situaciones de la vida real (Finkel, 2008). Estos temas generalmente se imparten de una forma independiente y no relacionada, dando una oportunidad de aplicar un ciclo de mejora que realce la utilidad de enseñar estos contenidos relativos al diseño de la edificación y afiance una mayor seguridad y éxito en el aprendizaje.

Para el diseño de este nuevo CIMA se parte de considerar que la metodología de clase invertida o "flipped classroom", podía contribuir a mejorar el conflicto teórico-práctico en la asignatura y demandar una mayor implicación y autonomía de los estudiantes, considerando en todo momento la forma de motivar y estimular los esfuerzos de los estudiantes mediante calificaciones parciales y valoración de la participación en el aula (Bergmann y Sams, 2012; Savery y Duffy, 1995). Este modelo de clase invertida contribuye a potenciar el razonamiento en grupo y la resolución de ejercicios prácticos teniendo en cuenta ejemplos y resoluciones que identifiquen los conceptos teóricos fundamentales a través de simulaciones en modelos virtuales y el análisis del comportamiento en la realidad (Strayer, 2012).

Sin embargo, para generar un modelo metodológico más eficaz y productivo entre la teoría y la práctica se ha considerado que es una buena oportunidad para 
combinar el modelo de clase invertida con la resolución de problemas, dando respuesta a retos y preguntas iniciales presentadas en el aula, que ayudara así a incrementar la activación y el interés del alumno por afrontar el aprendizaje de los contenidos seleccionados de una forma efectiva (Nolan y Washington, 2013; Boud y Feletti, 1997).

\section{Modelo metodológico}

El nuevo CIMA planteado para el Curso 2019 en la asignatura de Construcción I, en los bloques temáticos del confort lumínico, relacionados con el soleamiento, la iluminación natural interior y las técnicas de diseño pasivo en la envolvente del edificio pretende modificar la docencia desarrollada hasta el momento y combinar un aprendizaje basado en problemas, que dé respuesta a retos y preguntas iniciales para activar a los estudiantes y fomentar el interés y la participación, con un modelo docente basado en el aula invertida donde se priorice el tiempo de clase para dinamizar el razonamiento del grupo y potenciar el aprendizaje teórico y práctico a través de ejercicios resueltos y otros recursos complementarios. Por último, otro objetivo específico es el de interconectar y hacer ver la relación de tres bloques temáticos que hasta ahora han sido impartidos de una forma independiente y cuya comprensión sería mucho más eficiente desde una visión conjunta y entrelazada, como se percibe en la vida real.

La principal transformación del modelo metodológico que se introduce en el CIMA se basa en el concepto "Flipped classroom" o "Aula invertida" que supone como su propio nombre indica una inversión del trabajo docente y la comprensión asignados durante la clase y el tiempo anterior y posterior a la clase (Tucker, 2012). Para una comprensión directa y organizada se ha desarrollado el siguiente esquema (Tabla 1) que explica con mayor claridad el modelo metodológico planteado a través de una comparativa con 
el modelo tradicional, con el que se ha venido impartiendo la docencia durante los últimos años.

Tabla 1. Comparativa entre el modelo tradicional y el modelo de aula invertida implantado.

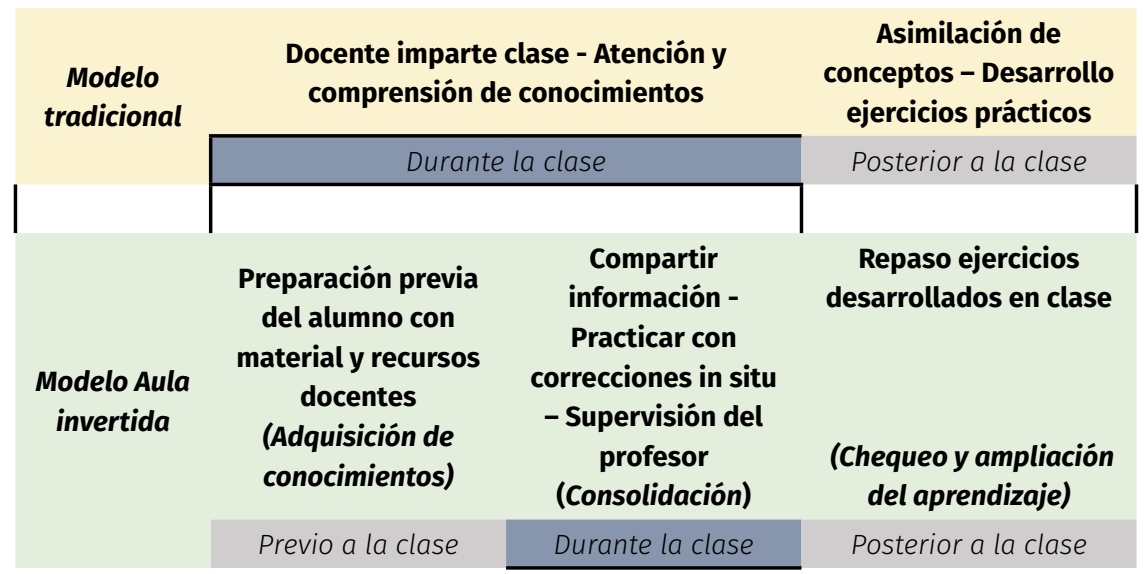

Este modelo metodológico combinado entre el aula invertida y el aprendizaje basado en problemas busca impartir clases mucho más interactivas donde el aprendizaje sea adquirido a través de un mayor razonamiento, aplicación a la realidad e interrelación de la utilidad entre bloques temáticos. Se presenta en la Figura 2 un esquema metodológico para definir la organización y estructura de la metodología diseñada. El esquema diferencia las actividades y objetivos perseguidos en las sesiones del aula y las actividades a desarrollar fuera de la misma, distinguiendo también las acciones y responsabilidades del profesor y de los alumnos.

Jornadas de Formación e Innovación Docente del Profesorado | № 2 (2019) Esta obra se distribuye con la licencia Creative Commons Reconocimiento-NoComercial-SinObraDerivada Internacional (CC BY-NC-ND 4.0.) 


\section{ANTONIO SERRANO-JIMÉNEZ}

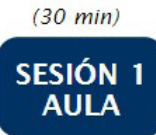

INTRODUCCIÓN PROFESOR

- Conceptos previos
- Objetivos del tema
- Organización de la
docencia
ALUMNOS
- Test inicial
- Dudas iniciales
grupanización en asignación
de modelos

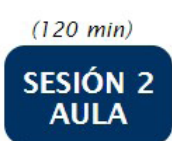

CONSOLIDACIÓN - PRÁCTICA

\section{FUERA DEL AULA}

CHEQUEO - AMPLIACIÓN

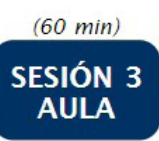

EVALUACIÓN - CIERRE
PROFESOR

$------------$

Selección del material

Recursos electrónicos

Motivar aprendizaje

ALUMNOS

- Visualización + Lectura

- Comprensión

- Anotación de dudas
ALUMNOS - PROFESOR

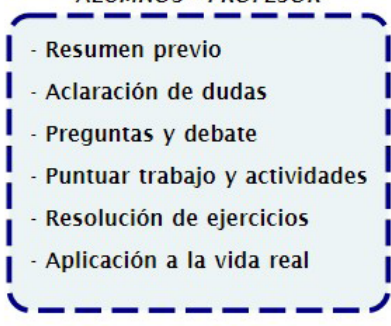

PROFESOR

- Diario de la sesión

- Ejercicios de ampliación

- Preguntas y respuestas surgídas en el aula

ALUMNOS

Í- Finalización de ejercicios

Ejercicios de ampliación

- Anotación de dudas

- Aclaración de dudas finales I

I - Debate de cierre final

- Reflexión sobre la utilidad I

Figura 2. Modelo metodológico propuesto en el CIMA.

Jornadas de Formación e Innovación Docente del Profesorado | № 2 (2019) 
Se describen a continuación las cinco fases fundamentales del modelo metodológico:

-1a Fase: Introducción. La metodología comienza con una introducción a los alumnos en la primera sesión, durante un periodo aproximado de entre 30 y 40 minutos, a modo de presentar los objetivos fundamentales del contenido a tratar, dar una explicación inicial sobre los principales conceptos previos necesarios y a explicar al alumnado cómo se va a organizar la docencia en este bloque temático y la utilidad de la misma, así como de realzar el valor extra en la evaluación que van a tener las actividades y el aprendizaje desarrollado fuera del aula. Esta introducción resulta necesaria para lanzar algunas preguntas abiertas o retos que van a estar presentes a lo largo de todo el ciclo para saber responder a las mismas y dar solución a estos problemas que se plantean, captando así el interés de los estudiantes y sirviendo de enlace para explicar el trabajo necesario a desarrollar fuera del aula previo a la segunda sesión. Finalmente, en los últimos minutos los alumnos harán un test inicial (Anexo I) para mostrar los conocimientos básicos previo adquiridos o que ya previamente poseían en el caso de alumnos repetidores, y la sesión se cerrará organizando los posibles grupos de trabajo.

-2a Fase: Aprendizaje. Se trata de una fase fundamental de la metodología planteada, pues la tarea del alumno fuera del aula es la de visualizar y comprender el material docente seleccionado por el profesor y expuesto en la plataforma virtual de trabajo de la clase. Se seleccionarán vídeos, tutoriales, apuntes, así como se introducirán ejercicios resueltos para facilitar la comprensión de los alumnos. La tarea para la próxima sesión será la de anotar dudas percibidas para que puedan ser resultas en clase, preferentemente en debates en grupo que cuenten con la moderación y el apoyo del profesor. Así pues, es una tarea fundamental para el profesor seleccionar el material 
teórico y los ejercicios para que pueda realizar un aprendizaje efectivo en los alumnos fuera del aula.

- 3a Fase: Consolidación - Práctica. Se trata de la sesión principal del bloque metodológico, que va a ser íntegra o que incluso, según la extensión del bloque de contenidos, podría ocupar dos sesiones. Contando con una base teórica expuesta en la sesión de introducción y en el aprendizaje adquirido por los alumnos, la sesión se presenta con un resumen inicial, la aclaración de las dudas anotadas por los alumnos, consideradas mediante preguntas o problemas abiertos y resueltos en debates y reflexiones en clase y la resolución de ejercicios prácticos de una forma individual o en grupo de trabajo durante la clase. Para resolver los ejercicios prácticos, los alumnos contarán con ejercicios similares resueltos, el material docente seleccionado por el profesor y el apoyo presencial del mismo profesor que podrá resolver las principales dudas surgidas en la resolución en clase, mostrando al mismo tiempo la aplicación real con la utilización de recursos online, fotografias, herramientas de visualización, etc.

- 4a Fase: Chequeo - Ampliación. Se trata de una fase a desarrollar fuera del aula donde los alumnos llevarán a cabo la finalización de los ejercicios y tendrán la opción de resolver otros ejercicios de ampliación expuestos por el profesor. Entre tanto, el profesor tendrá la tarea de anotar en un diario de la sesión llevada a cabo las principales preguntas y respuestas surgidas en el aula, las principales utilidades de los ejercicios desarrollados y seleccionar en la plataforma los posibles ejercicios de ampliación.

- 5á Fase: Evaluación - Cierre. En una tercera sesión, o sesión final, la sesión comenzará con una tarea de corrección y evaluación intercambiando los ejercicios 
desarrollados entre los mismos alumnos. Las correcciones se realizarán en función del conocimiento de los alumnos y el asesoramiento en todo momento del profesor. Además, será el momento apropiado para aclarar las principales dudas surgidas y ofrecer explicaciones finales sobre la resolución de ejercicios prácticos y el significado de los principales contextos. Para finalizar, se llevará a cabo un debate final sobre la utilidad del contenido tratado y la relación con los otros bloques temáticos, así como se desarrollará el mismo test inicial para la evaluación final (Anexo I) para que el profesor pueda evaluar el conocimiento adquirido con respecto al conocimiento inicial.

Se presenta a continuación un mapa de los contenidos organizadores abordados en el CIMA (Figura 3), así como los problemas a los que cada bloque responde, para ofrecer así una relación global del ciclo de mejora y los contenidos a tratar durante las sesiones. Los tres bloques temáticos presentados tendrán una secuencia similar siguiendo el modelo metodológico propuesto y en su totalidad, la aplicación del CIMA en el aula será desarrollada en 12 horas, organizadas en seis sesiones de dos horas cada una.

Con este modelo se pretende hacer una clase mucho más dinámica e interactiva, en la cual el alumnado pueda participar con facilidad, preguntar y aclarar aquellas dudas que le surjan a fin de afianzar los conceptos básicos. En definitiva, este modelo metodológico consigue cambiar una clase que estaba claramente dividida entre la teoría y la práctica, pasando a ser una combinación teórico-práctica, en la cual mientras se da respuesta a preguntas, retos o problemas iniciales y se enseñan los conceptos teóricos fundamentales a través de la resolución práctica de ejercicios en el aula con el apoyo de sus compañeros y el profesor. 
Cómo orientamos nuestro edificio?

¿Qué influye el diseño de volúmenes en las sombras arrojadas?

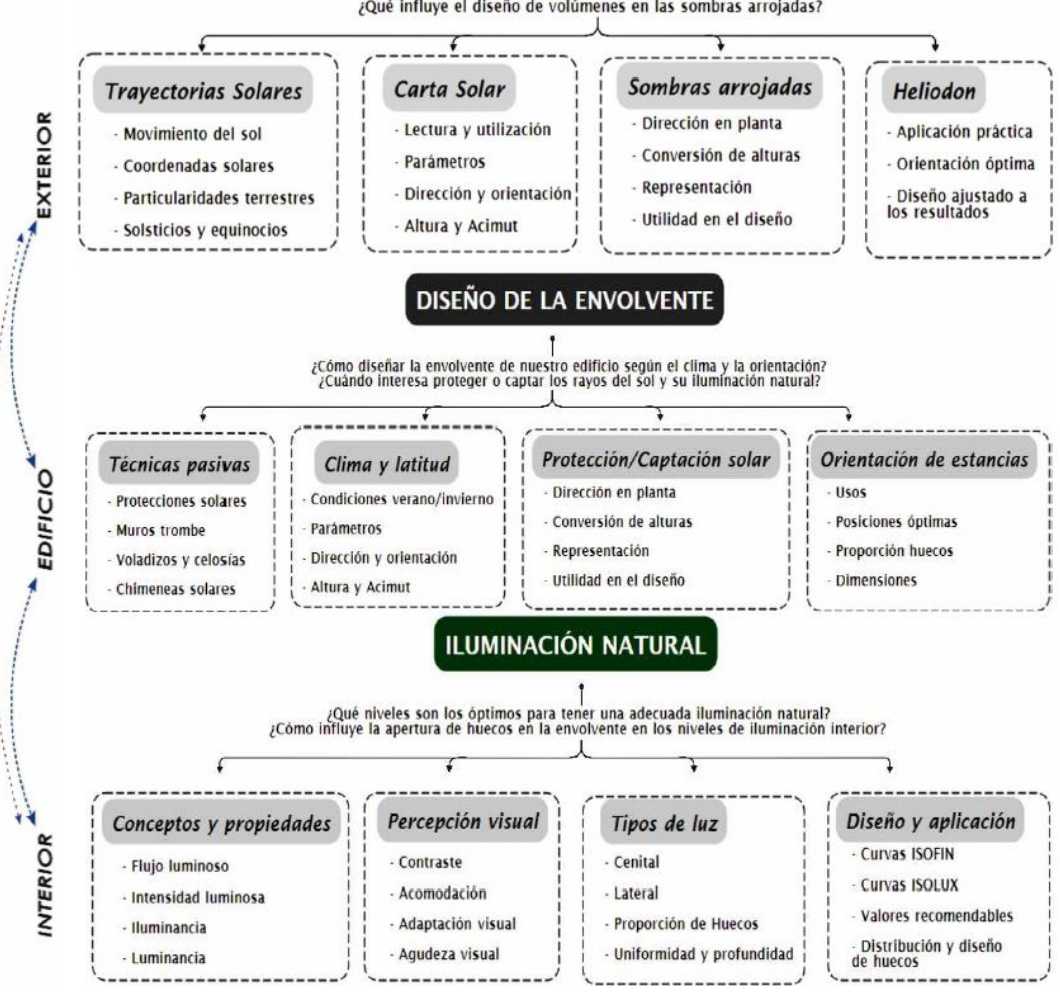

Figura 3. Mapa de los contenidos organizadores propuesto en el CIMA.

\section{Aplicación CIMA}

Tras presentar el modelo metodológico propuesto para el CIMA y los contenidos seleccionados del temario de la asignatura de Construcción 1 donde aplicarlo, se detallan a continuación la secuencia de actividades llevadas a cabo en las seis sesiones y en el trabajo fuera del aula entre dichas sesiones, que han constituido el conjunto de tareas para conseguir cumplir con el CIMA diseñado.

- Sesión 1. Presentación - Soleamiento. La sesión comenzó con la presentación a los alumnos del modelo metodológico que se iba a aplicar y los bloques

Jornadas de Formación e Innovación Docente del Profesorado | № 2 (2019) Esta obra se distribuye con la licencia Creative Commons Reconocimiento-NoComercial-SinObraDerivada Internacional (CC BY-NC-ND 4.0.) 
de contenido que se van a impartir a lo largo de las siguientes sesiones, así como el sistema de evaluación que se iba a considerar, explicando que se tendría mucho en cuenta la participación e integración de los alumnos en la dinámica de la clase. El planteamiento de las cuestiones fundamentales del tema, en preguntas abiertas y generando debates con los alumnos, condujo al profesor a introducir los conceptos previos del soleamiento, los parámetros fundamentales y la utilidad que representa en el diseño de los edificios a la hora de situar, diseñar la envolvente y optimizar este recurso natural lumínico. Además, se llevó a cabo durante 15 minutos el test inicial detallado en el Anexo I, con las preguntas fundamentales del conjunto de contenidos. Finalmente se explicó a los alumnos las tareas a desarrollar fuera del aula para la siguiente sesión, el modo de acceder al material docente y digital que fue subido a la plataforma de clase y los objetivos que persiguen para la siguiente sesión.

- Trabajo fuera del aula (Aprendizaje - Soleamiento). Esta fase fue una parte fundamental de la metodología implantada ya que los alumnos, de una forma individual o en grupo, debían visualizar fuera del aula el material seleccionado por el profesor para adquirir la utilidad y aplicación de los conceptos fundamentales del soleamiento previo al desarrollo del ejercicio práctico en la siguiente sesión. Para ello se seleccionaron vídeos explicativos, modelos tridimensionales y otros recursos electrónicos. Los alumnos tuvieron como tarea la visualización o lectura de este material y adquirir la máxima comprensión posible apuntando el conjunto de dudas e incomprensiones surgidas para resolverlas al día siguiente en clase. Los conceptos previos ofrecidos por el profesor en la sesión 1 unido al material docente afrontado por los alumnos constituyó un aprendizaje inicial que conforma una base de 
conocimiento para la resolución práctica de ejercicios en la siguiente sesión.

- Sesión 2. Soleamiento. Se trata de la sesión más característica de la metodología de clase invertida, pues los alumnos llegaron a clase con un conocimiento previo adquirido de la sesión 1 y del aprendizaje desarrollado fuera del aula y pasaron a resolver ejercicios prácticos en clase, en grupos de trabajo, con el apoyo del profesor para resolver las principales dudas surgidas. Inicialmente se presentó un resumen de la clase inicial por parte del profesor, apoyado por las respuestas a las dudas surgidas por los alumnos y a las explicaciones generadas en debates a las preguntas abiertas planteadas. A continuación, diferentes grupos de alumnos pasaron a resolver diferentes modelos de ejercicios prácticos, para representar las sombras arrojadas en diferentes horas y días del año, lo que llevó a aprender y practicar los procedimientos de conversión de alturas, identificación de la posición del sol y obtención de la dirección de la sombra. Para su resolución tuvieron, además de la presencia del profesor, el apoyo de ejercicios resueltos entregados por el profesor (Figura 4), para ver el procedimiento y los objetivos que se persiguen en su resolución, así como se debatió sobre las implicaciones que supone el conocimiento de estos ejercicios en el diseño del edificio, su orientación y emplazamiento y su aplicación en la vida real. 

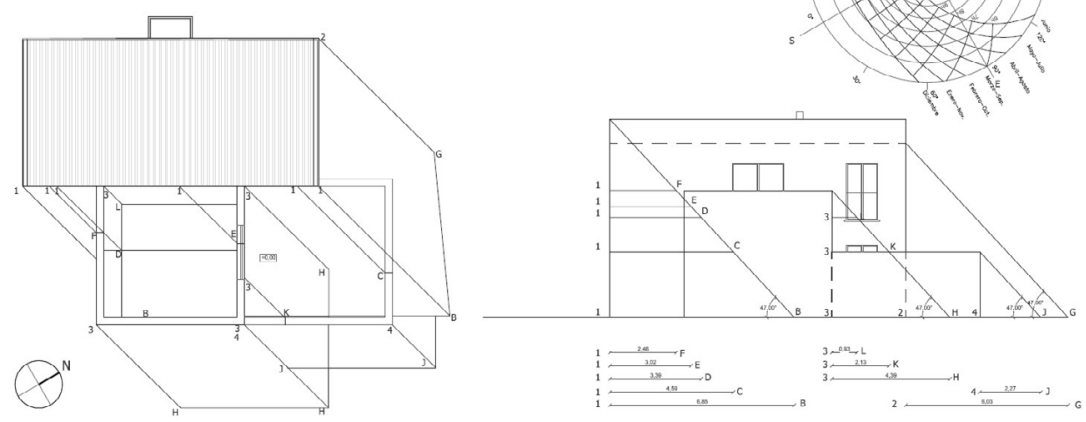

Figura 4. Ejemplo de ejercicio resuelto de sombras arrojadas entregado por el profesor.

- Trabajo fuera del aula (Ampliación - Soleamiento). El trabajo de los alumnos fuera del aula tras la segunda sesión fue el de finalizar el ejercicio práctico para su entrega en la próxima sesión ya que no hubo tiempo suficiente para que los alumnos lo finalizasen en el aula. Además, el profesor se encargó de redactar un pequeño diario de la sesión con las principales dudas detectadas durante la resolución de ejercicios y comprensión de conceptos, con las respuestas que se dieron y los debate generados. También incluyó en la plataforma nuevos ejercicios de ampliación. Estos ejercicios de ampliación buscaron además mostrar la utilidad en el diseño al representar las sombras arrojadas, como la situación de una piscina en una urbanización. Por último, en esta fase los alumnos también deberán anotar las dudas e incomprensiones surgidas.

- Sesión 3. Soleamiento - Iluminación natural. Esta tercera sesión se centró en la evaluación y cierre del ciclo aplicado al bloque de contenidos de Soleamiento. Los alumnos repartieron las prácticas 
finalizadas entre sus compañeros aleatoriamente y se realizó una corrección conjunta en base a las explicaciones del profesor y los conocimientos adquiridos por los compañeros. Además, se dedicó un tiempo de la clase a resolver las dudas finales, a generar un debate entre profesor y alumnos para analizar la utilidad, la aplicación a la vida real y su influencia en el diseño arquitectónico. Por otro lado, la segunda mitad de esta sesión enlazó con la sesión inicial del bloque de contenidos de lluminación natural, siguiendo la semejanza de organización explicada en la sesión 1, con el planteamiento de las preguntas iniciales, la explicación de los conceptos previos y la organización del bloque de contenidos dentro del mismo modelo metodológico. El ejercicio práctico de iluminación natural se planteó sobre el mismo edificio en el que se han resuelto las prácticas de soleamiento.

- Trabajo fuera del aula (Aprendizaje - Iluminación natural). Los alumnos tuvieron que visualizar y comprender el material docente relacionado con el bloque de contenidos de lluminación natural. Se buscaba que comprendieran en casa los parámetros fundamentales que cuantifican e influyen en el diseño para ofrecer una adecuada iluminación natural en el interior de los espacios, así como conocer los parámetros orientativos para los que se encaja el diseño aconsejable de espacios en función de su uso y la clasificación fundamental del tipo de iluminación.

- Sesión 4. Iluminación natural. La cuarta sesión estuvo enfocada en consolidar el aprendizaje adquirido por los alumnos tanto fuera del aula como en la sesión inicial de este bloque, de saber responder a las preguntas o problemas iniciales y de realizar diversos ejercicios prácticos basados en modelos expuestos por el profesor, para analizar los valores y resultados de iluminación y otros factores influyentes 
en distintos escenarios. Se buscó que los alumnos reflexionasen en base a ejercicios ya desarrollados analizando la influencia de colocar diversos huecos, con diferentes dimensiones y situaciones, y la incidencia que tiene en los resultados finales de iluminación la posición de los huecos en un espacio. También se debatió sobre la idoneidad que requieren los diferentes usos edificatorios en las pautas de diseño para el control de la iluminación natural. Los alumnos trabajaron en clase en un informe individual que lo pudieron terminar fuera del aula para aquellos que no terminaron durante el tiempo de clase.

- Trabajo fuera del aula (Ampliación - Iluminación natural). Los alumnos tuvieron que finalizar el informe final sobre los modelos entregados en clase y el análisis y reflexión de los resultados y la influencia en el diseño de la envolvente. De nuevo el profesor se encargó de redactar un pequeño diario de la sesión con las principales dudas surgidas durante la resolución de ejercicios y comprensión de conceptos, con las respuestas y el debate generado. También incluyó en la plataforma nuevos ejercicios de ampliación.

- Sesión 5. Iluminación natural - Diseño pasivo. La quinta sesión se destinó a hacer una corrección de los ejercicios relativos a la iluminación natural y a debatir sobre el aprendizaje adquirido en estas sesiones. También se atendieron las dudas fundamentales surgidas por los alumnos en el análisis de las soluciones y los parámetros tenidos en cuenta de los modelos utilizados. Esta sesión pasó a introducir también el bloque de contenidos de acondicionamiento pasivo, más reducido en cantidad, pero altamente relacionado con los bloques temáticos impartidos.

- Trabajo fuera del aula (Aprendizaje - Diseño pasivo). Los alumnos hicieron uso del material 
docente colgado, en vídeos y tutoriales, para comprender las técnicas principales del diseño pasivo y las implicaciones que suponen en el ahorro energético y el aprovechamiento, a través del diseño de la fachada, de los recursos naturales del entorno y diferenciar las condiciones de verano e invierno en diferentes zonas climáticas. Estos conceptos serían chequeados y reforzados en la sesión final.

- Sesión 6. Diseño pasivo. La sesión final sirvió para responder y resolver ejercicios cortos sobre las pautas de diseño pasivo en edificios situados en diferentes localizaciones y en diferentes condiciones de invierno o verano. Estas soluciones fueron debatidas en clase y se trató de interrelacionar el aprendizaje adquirido en este CIMA, con un debate que sirvió como cierre final. Por último, se destinó la parte final de la clase a resolver el test final para valorar el aprendizaje adquirido por los alumnos y a volver a responder al conjunto de preguntas o retos iniciales planteados, una vez adquirido el conocimiento.

\section{Evaluación del CIMA y principios didácticos incorporados}

El aprendizaje adquirido por los estudiantes con la innovación diseñada en el CIMA ha sido evaluado a través de los resultados obtenidos en el test inicial y final sobre los bloques temáticos seleccionados, así como evaluar la calidad en el razonamiento de los alumnos en las respuestas. Para la valoración de los resultados se ha recurrido a escaleras de aprendizaje que secuencian las diferentes fases de aprendizaje de los alumnos en las respuestas ofrecidas y la resolución de los ejercicios, como puede verse en el siguiente esquema con resultados correspondientes al porcentaje de alumnos situados según el test inicial y final (Figura 5). 

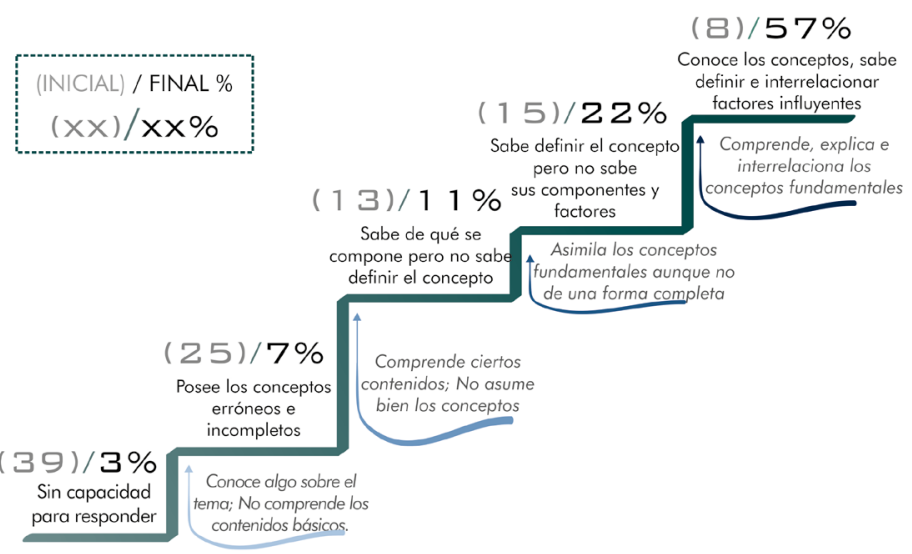

Figura 5. Escalera de evaluación de conocimientos con resultados del test inicial y final.

Los resultados obtenidos muestran la efectividad del modelo metodológico, demostrando que en torno al $80 \%$ de los alumnos adquieren los contenidos fundamentales y el procedimiento de resolución de numerosos ejercicios prácticos del bloque de contenidos. Además, se ha incorporado en este modelo metodológico un nuevo bloque de evaluación que ha valorado el esfuerzo y trabajo desarrollado por los alumnos fuera y dentro del aula, estimulando la participación y las explicaciones en el aula por parte de los alumnos al aclarar dudas o incorporar nuevas explicaciones.

Así pues, la calificación obtenida en los ejercicios prácticos forma parte de la calificación de la parte práctica de la asignatura, con un porcentaje a priori asignado del $40 \%$ en su totalidad, así como se ha asignado una valoración extra en aquellos alumnos que han participado en los debates y en la resolución de dudas de los compañeros, con un porcentaje asignado del 5\% del total de la asignatura, fomentando y motivando así la participación en clase para llevar a cabo un aprendizaje comunitario.

Respecto a los principios didácticos introducidos, el CIMA ha incluido una serie de patrones o regularidades esenciales a la hora de establecer el nuevo modelo de 
enseñanza, teniendo en cuenta los contenidos establecidos, el estilo de aprendizaje deseado y los recursos generados para llevarlo a cabo. Se detallan a continuación los principales principios didácticos establecidos:

- Clase invertida. Se han intercambiado algunas tareas de aprendizaje que previamente se desarrollaban fuera del aula, a su resolución durante el tiempo de clase, contando con la experiencia y el apoyo del profesor para facilitar y potenciar la adquisición de los conocimientos fundamentales a través del estudio y análisis de ejercicios resueltos o la visualización del comportamiento en las simulaciones de diferentes escenarios, lo que ha aumentado el tiempo de clase práctica y la resolución de problemas en el aula.

- Resolución de problemas. Se ha otorgado una gran importancia a la comunicación abierta y dialogante en clase entre el profesor y los alumnos, con la incorporación de preguntas abiertas, problemas o retos, que generen la participación de los alumnos, fomentar debates en clase con el razonamiento de diferentes respuestas y, además, ha existido también un especial interés en hacer perder a los alumnos el miedo a equivocarse, sobre todo al ser alumnos de primer curso recién accedidos a la universidad.

- Autonomía del aprendizaje en el aula. Se tratado de promover que el alumno adquiera una autonomía del aprendizaje en clase, en contra de hacer la reflexión sobre la teoría y la aplicación a la práctica en casa, como venía haciendo hasta ahora. Esta autonomía del aprendizaje vendrá motivada por la comprensión de los conceptos teóricos con la resolución y análisis de las soluciones de ejercicios prácticos de forma individual o en grupo.

- Aplicación a la realidad. Se ha pretendido que la aplicación a circunstancias que se dan en nuestro día a día y la definición de ejemplos sea una constante en el desarrollo de las sesiones, permitiendo 
a los alumnos reconocer diversos abstractos en situaciones comunes del día a día y a su vez captar la atención para conseguir un aprendizaje mucho más efectivo.

- Recursos y herramientas digitales. Se ha incorporado numerosos recursos digitales, que, añadidos a los recursos materiales utilizados con anterioridad, facilitan la comprensión y visualización de soluciones en ejercicios prácticos a través de vídeos, webs o herramientas informáticas que son susceptibles de integrar en la nueva metodología docente.

- Evaluación en el aula y progresión del aprendizaje. Se implementará la opción de corregir y evaluar los ejercicios entre los mismos alumnos durante el tiempo de clase, que permita razonar la resolución de los mismos entre compañeros, con el apoyo del profesor. Además, para una valoración docente se han desarrollado escaleras de aprendizaje que chequeen el estado inicial y final con la utilización de un cuestionario inicial y final.

El profesor ha querido obtener una evaluación de la metodología docente implantada ofreciendo a los alumnos rellenar un breve cuestionario sobre la satisfacción, adecuación y reseña de los aspectos negativos y positivos del nuevo modelo docente. Los resultados han sido en general muy satisfactorios, con una satisfacción muy elevada entre los alumnos, marcando todos ellos la idoneidad del nuevo modelo y proponiendo algunas mejoras como el dar más tiempo para las entregas de los ejercicios prácticos o planificar la visita a alguna obra dentro de la asignatura.

Por último, aunque se han encontrado diversos escollos y barreras a la hora de implicar a los alumnos y aumentar la participación en el aula, la organización de los bloques temáticos alrededor de preguntas (Resolución de problemas) y la mayor dedicación de tiempo en el aula 
para resolver ejercicios prácticos gracias a nuevos recursos y ejemplos resueltos (clase invertida) ha generado una mayor satisfacción en el profesor para la aplicación de los contenidos seleccionados, contribuyendo a que sea un uso habitual en sus sesiones docentes de estas asignaturas técnicas del Grado de Fundamentos de la Arquitectura.

Jornadas de Formación e Innovación Docente del Profesorado | № 2 (2019) Esta obra se distribuye con la licencia Creative Commons 


\section{Referencias}

Bain, K. (2007). Lo que hacen los mejores profesores universitarios. Valencia: Publicaciones de la Universidad de Valencia.

Bergmann, J. y Sams, A. (2012). Flip your classroom: Reach every student in every class every day". International Society for Technology in Education. Washington DC.

Boud, D. y Feletti, G. (1997). The challenge of problem-based learning. Londres: Psychology Press.

Finkel, D. (2008). Dar clase con la boca cerrada. Valencia: Publicaciones de la Universidad de Valencia.

Krauss, J. y Boss, S. (2013). Thinking through Project-based learning: Guiding deeper inquiry. California: Corwin - A SAGE company.

Nolan, M.A. y Washington, S.S. (2013). Flipped out: Successful strategies for improving student engagement.

Savery, J.R. y Duffy, T.M. (1995). "Problem-based learning: An instructional model and its constructivist framework" en Educational Technology, vol. 35, n으, p. 31-38.

Serrano-Jiménez, A., Barrios-Padura, Á y Molina-Huelva, M. (2017). "Towards a feasible strategy in Mediterranean building renovation through a multidisciplinary approach" en Sustainable Cities and Society, vol. 32, p. 532-546.

Strayer, J.F. (2012). How learning in an inverted classroom influences cooperation, innovation and task orientation". Learning Environments Research, vol. 15, no. 2, 171-193.

Tucker, B. (2012). The flipped classroom, Education Next, vol. 12, no. 1, pp.82- pp.83.

Jornadas de Formación e Innovación Docente del Profesorado | № 2 (2019) Esta obra se distribuye con la licencia Creative Commons 
Anexo I

\section{CONSTRUCCIÓN 1. CURSO 2019-2020 TEST INICIAL/FINAL}

1. [Carta solar _ Conceptos] Para la carta solar de Sevilla, di en qué día o días del año y en qué momento/s del día el sol se sitúa lo más al norte posible.

2. Para la carta solar del ecuador. ¿Qué acimut y altura tendría en el equinoccio a las $12 \mathrm{~h}$ del mediodía? ¿Qué sombra arrojada tendría un edificio de 3 plantas de altura?

3. [Sombras arrojadas _ Aplicación práctica] Proyecta a mano alzada la sombra que tendría el siguiente volumen situado en Sevilla en el solsticio de verano a las 10 horas.

4. [Iluminación natural _ Interpretación gráfica] Analiza el siguiente gráfico y explícalo indicando qué factor y/o unidades se están representando, así como la idoneidad de las mismas respecto a los valores aconsejados. En caso de ser insuficiente, ¿de qué forma modificarías el diseño?

5. [Diseño pasivo _ Aplicación de conceptos] ¿En una ciudad situada en Noruega, explica qué técnicas y sistemas de acondicionamiento pasivo emplearías para el diseño de su envolvente? ¿Y si fuera en una ciudad del sur de Grecia? Cita algunos ejemplos.

Jornadas de Formación e Innovación Docente del Profesorado I № 2 (2019) Esta obra se distribuye con la licencia Creative Commons Reconocimiento-NoComercial-SinObraDerivada 4.0 Internacional (CC BY-NC-ND 4.0.) 